\title{
Indications for vitrectomy in congenital retinoschisis
}

\author{
JOEL SCHULMAN,'? GHOLAM A PEYMAN,' NORBERT JEDNOCK' \\ AND BRUCE LARSON' \\ From the Departments of Ophthalmology, 'University of Illinois at Chicago, Eye and Ear Infirmary, and the \\ 'University of Texas Medical Branch, Galveston, Texas
}

SUMmaRY Two patients with congenital retinoschisis developed rhegmatogenous retinal detachments with either a tractional component or associated vitreous haemorrhage. The second patient also had a large, raised schisis cavity under vitreous traction, which obscured visualisation of the macula. Vitrectomy is indicated for some patients with congenital retinoschisis.

Congenital retinoschisis, a relatively rare ocular disorder' first described by Haas' in 1898 , has also been called congenital vascular veils, ${ }^{3}$ cystic disease of the retina in children, ${ }^{+}$juvenile retinoschisis, ${ }^{5}$ and inherited retinal detachment. ${ }^{\circ}$ Usually bilateral, ${ }^{7}$ the disorder is inherited as an X-linked Mendelian recessive trait affecting males, ${ }^{,-11}$ though a few sporadic cases have been reported in females."

Retinoschisis has a predilection for the inferotemporal quadrant ${ }^{7}$ and rarely extends to the ora serrata. Large blood vessels frequently course through the inner retinal wall, and other vascular elements may traverse the schisis cavity.' Retinal holes in the inner layer are common and usually appear in multiples. ${ }^{13}$

Other ocular changes reported in retinoschisis include neovascular glaucoma, ${ }^{14}$ optic atrophy, ${ }^{515}$ and diminished visual acuity ${ }^{16}$ secondary to foveal dystrophy. Conway and Welsh'2 described the presence of a haemorrhagic retinal cyst surrounded by exudate extending into the macula.

Retinal detachment is a serious complication associated with congenital retinoschisis. ${ }^{57131718}$ Surgical treatment in the past has involved conventional scleral buckling procedures. The use of vitrectomy to remove vitreous haemorrhage and to repair a retinal detachment with a tractional component in patients with $\mathrm{X}$-linked retinoschisis is, to the best of our knowledge, described for the first time here.

\section{Case reports}

CASE 1

An 11-year-old boy, who was diagnosed as having $X$ Correspondence to Dr Gholam A Peyman, 1855 W Taylor St, Chicago, IL 60612, USA. linked retinoschisis at age 5, was referred to the University of Illinois Eye and Ear Infirmary with a retinal detachment in his right eye. He was the product of a normal pregnancy and had no congenital anomalies. Two older brothers also had X-linked retinoschisis. An ocular examination of his mother and three of his sisters was unremarkable. No consanguinity existed between the parents. When the patient was 4 years old, an electroretinogram (ERG) (performed with the patient under anaesthesia) showed an extinguished B wave. The boy's two brothers had similar ERG changes.

Ocular examination revealed a corrected vision of counting fingers at 6 feet $(1.8 \mathrm{~m})$ in the right eye and $5 / 200$ in the left eye. External examination revealed $8^{\circ}$ of left esotropia. Biomicroscopy was unremarkable. Ophthalmoscopy of the left eye revealed a large grey intravitreal membrane floating in the inferior vitreous between the inferior arcade and equator. The macula was distorted, and inferiorly a retinoschisis cavity was observed. A fundus examination of the right eye disclosed cystic macular changes. A fullthickness retinal detachment (Fig. 1) that involved the macula and most of the inferior retina extended from the 4 to 8 o'clock meridians. A small schisis cavity was noted in the inferior retina near the equator. Another area of retinoschisis was present inferior to the temporal arcades. A vitreous veil exerted traction on a retinal fold along the inferotemporal arcade. A partial-thickness retinal tear was also present below the retinal fold.

During surgery the partial-thickness retinal tear inferior to the macula was noted to be under considerable vitreous traction. The vitreous band was transsected with a bipolar ${ }^{19}$ cautery attached to the fiberoptic membrane dissector and vitrophage to allow the underlying retina, including the partial- 


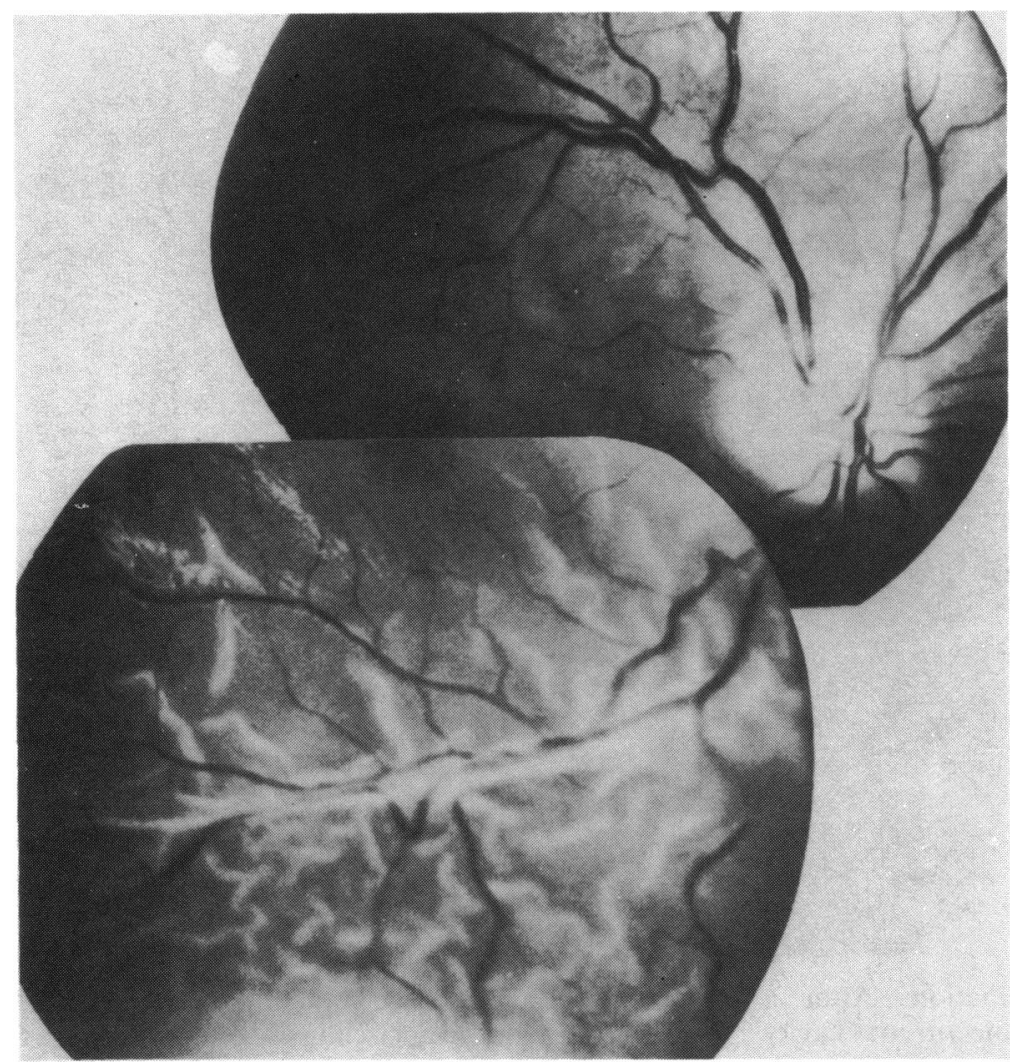

Fig. 1 Case 1. Composite fundus photograph showing flat detached retina inferiorly and in the macula.

thickness retinal tear, to settle. The inferior peripheral retina settled during the completion of the vitrectomy. Peripheral transscleral cryopexy was applied to the eye for $360^{\circ}$.

Postoperatively the retina remained attached (Fig. 2). At last examination seven months after surgery the best corrected visual acuity was 20/200.

\section{CASE 2}

A 2-year-old black boy had a red left eye that was diagnosed as viral conjunctivitis. On follow-up examination three weeks later the child was found to have a preretinal haemorrhage in the right eye and a possible retinal detachment in the left eye.

He was living in a foster home with his 3-year-old brother and no family history was available. Ocular examination of his brother was unremarkable.

Five weeks after his initial presentation the boy was admitted to hospital for an examination under anaesthesia. The right eye showed stellate cystoid changes in the macula and extensive retinoschisis in the inferior and temporal periphery, extending centrally with abrupt termination at the posterior pole. The left eye had a highly elevated bullous schisis cavity inferiorly overhanging and obscuring visualisation of the macula (Fig. 3). A second area of schisis was present in the superior temporal area.

The patient was again examined under anaesthesia six weeks later and underwent drainage of schisis fluid, vitrectomy to relieve traction on the inferior schisis cavity, and placement of an encircling silicone band in the left eye. Stellate cystoid changes were observed in the left macula after the schisis cavities were flattened.

He was readmitted for an examination under anaesthesia 10 months later. Examination of the left eye revealed a reaccumulation of fluid in the inferior and temporal schisis cavity, which again covered the macula. Three months later the child underwent surgery in the left eye, which involved drainage of fluid from both schisis cavities, and then scattered transscleral cryocoagulation was applied to the now flattened inferior and temporal schisis cavity. Twenty months after operation the retina remained attached (Fig. 4).

Nineteen months after the initial admission to hospital a vitreous haemorrhage (Fig. 5) and an inferior retinal detachment were discovered in the 
Fig. 2 Case 1. Fundus photograph after vitrectomy and retinal detachment repair.

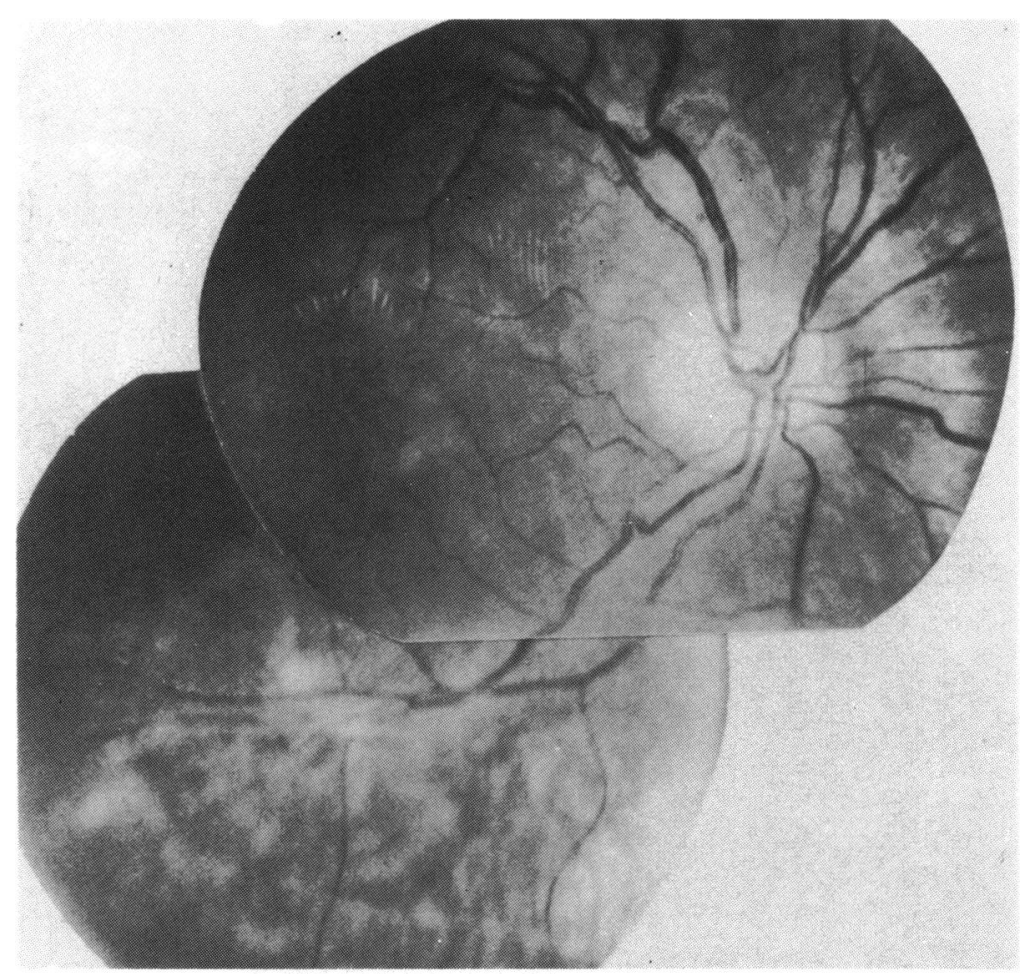

right eye during routine examination. After a vitrectomy to remove blood from the vitreous cavity two large full-thickness retinal tears were observed. Subretinal fluid and fluid in the large schisis cavity

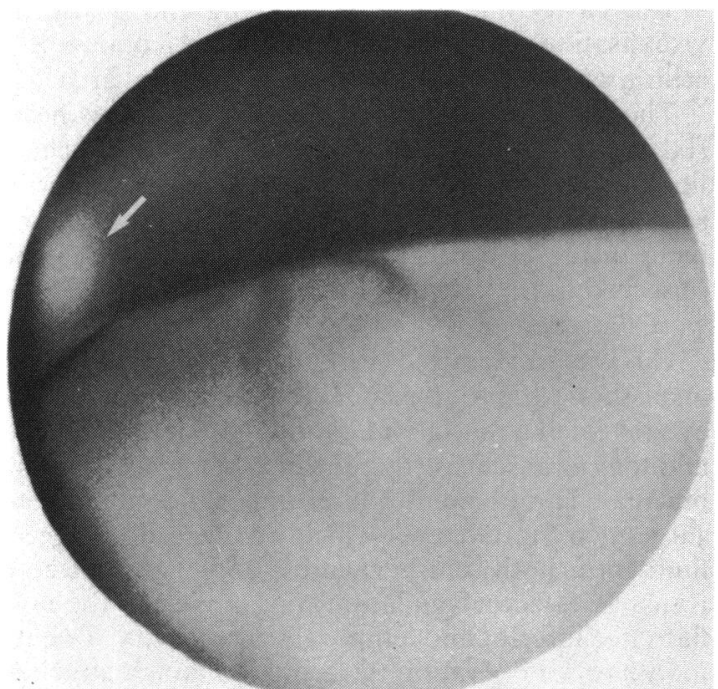

Fig. 3 Case 2. Left eye fundus photograph showing huge schisis cavity covering the macular area. Arrow points to optic disc. was drained, and the two retinal tears were treated with cryopexy and an encircling silicone band, and a 5 $\mathrm{mm}$ radial silicone sponge was placed behind both retinal tears.

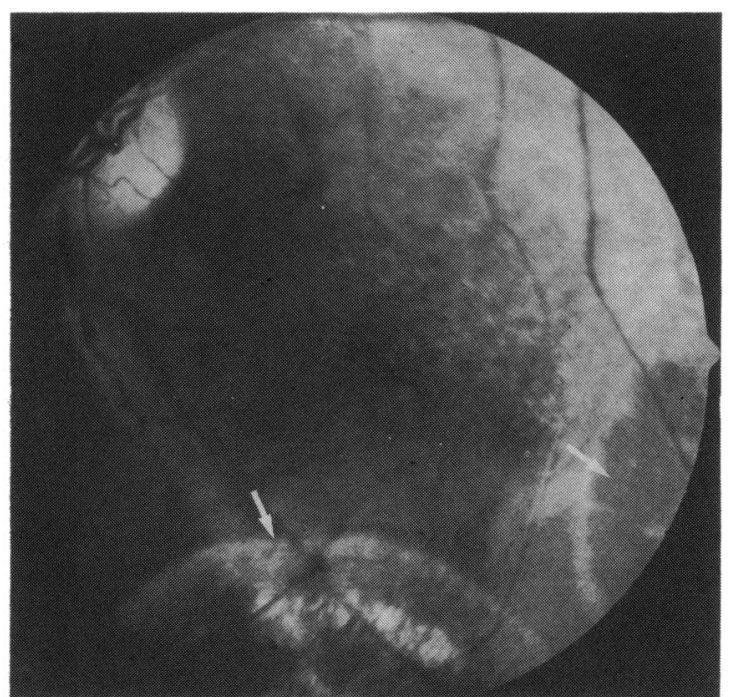

Fig. 4 Case 2. Fundus photograph after vitrectomy, retinal detachment repair, and cryocoagulation. Note attached retina and typical schisis changes of the macula; arrows indicate cryocoagulation scar. 


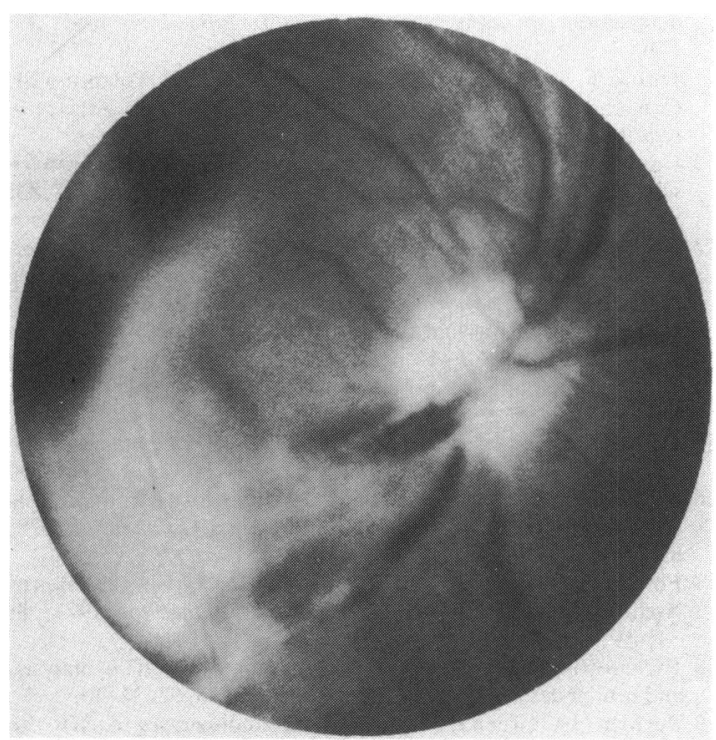

Fig. 5 Case 2. Fundus photograph of right eye showing vitreous haemorrhage covering a detached inferior retina.

Postoperatively a cataract developed in the right eye, which required a lensectomy a year later. At the time of the cataract surgery the retina and schisis cavities were noted to be flat (Fig. 6). Measurement of visual acuity was not possible because of the patient's youth.

\section{Discussion}

Congenital retinoschisis may be progressive or stationary or may spontaneously regress. ${ }^{13}$ The clinical spectrum of ocular changes varies considerably among affected individuals. Deutman ${ }^{20}$ states that macular retinoschisis occurs in all affected patients. Foveal retinoschisis in one report was present in $98 \%$ of involved individuals. ${ }^{21}$ According to Deutman ${ }^{21}$ in almost half the affected eyes macular lesions may represent the only sign of this disorder. The macular changes in older people may appear atrophic and pigmented. ${ }^{22-24}$ Peripheral retinoschisis, characterized by intraretinal splitting involving the nerve fibre layer, ${ }^{25}$ occurs in approximately $50 \%$ of eyes. ${ }^{26}$ The inner layer is often immobile and elevated in a concave configuration. An absolute scotoma is present corresponding to the area of retinoschisis. ${ }^{25}$

Two severe vision-threatening complications of congenital retinoschisis are retinal detachment and vitreous haemorrhage. ${ }^{2}$ Retinal detachments are caused by a combination of inner and outer layer holes in areas of retinoschisis, or retinal tears created by vitreous traction in abnormal retina. ${ }^{13}$ Vitreous haemorrhage may result from vitreous traction on

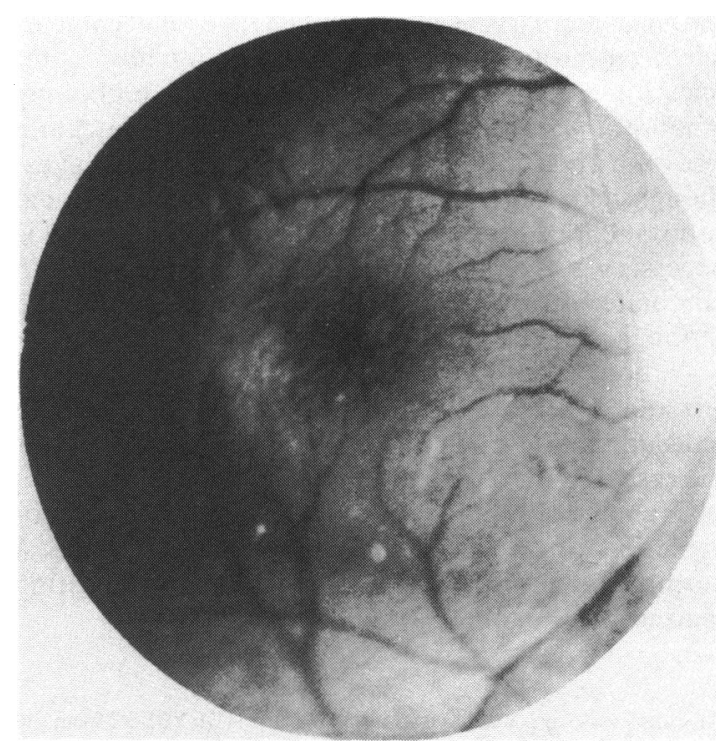

Fig. 6 Case 2. Same eye as in Fig. 5 after vitrectomy, retinal detachment repair, and buckling procedure.

retinal vessels coursing through the elevated inner retinal layer of a schisis cavity.

A variety of vitreous pathology has been observed in eyes with congenital retinoschisis. All 15 patients in a series reported by Lisch ${ }^{26}$ showed varying degrees of vitreous liquefaction. Vitreous strands were found in $50 \%$ of patients by $\mathrm{Bec}$ and associates. ${ }^{27} \mathrm{Kraushar}$ et al. ${ }^{13}$ reported a series of 40 patients ( 77 eyes) with congenital retinoschisis. Retinal detachment developed in $22 \%$ of these affected eyes. Associated with retinal detachment were pathological vitreous changes, including vitreous membranes attached to and in some instances elevating the equatorial retina. Vitreous traction present in the posterior pole resulted in pseudopapilloedema (13\% of eyes) and ectopic macula ( $6 \%$ of eyes). Vitreous haemorrhage occured in $40 \%$ of eyes. The investigators believed that in some instances vitreous haemorrhage was caused by traction on retinal blood vessels. Other less common signs of vitreous traction were tenting of the inner retinal layer $(12 \%)$ or blood vessels $(4 \%)$ and geographic areas of white without pressure $(35 \%)$.

The retinas in both our patients and the inferior schisis cavity overhanging and obscuring visualisation of the macula were under vitreous traction. The retinas in both cases were successfully reattached following vitrectomy and scleral buckling. Vitrectomy in eyes with congenital retinoschisis is indicated when a significant tractional component is associated with either retinal detachment, elevation of a schisis cavity covering the macula, or vitreous haemorrhage originating from blood vessels located in the inner 
retinal layer of a schisis cavity. The tractional changes may result from proliferative vitreoretinopathy ${ }^{28}$ complicating a retinal detachment or vitrous pathology inherent in congenital retinoschisis. Scleral buckling does not treat the vitreous pathology in these eyes, though it may relieve to some extent the tractional component of the retinal detachment. Vitrectomy is necessary to remove vitreous haemorrhage and to eliminate vitreous traction on the vessels and the retina.

Although vitrectomy prevents progression of vitreous tractional changes, it will not eliminate re.iccumulation of fluid in the schisis cavity, as was the case in our second patient.

Drainage of subretinal fluid combined with cryocoagulation of the suspected retinal holes creates a permanent chorioretinal scar, thereby preventing reaccumulation of fluid in the schisis cavity.

This work was supported in part by core grant 1P3EY01792 from the National Eye Institutc, Bethesda, Md, USA.

\section{References}

1 Mann I, MacRae A. Congenital vascular veils in the vitreous. $\mathrm{Br}$ J Ophthalmol 1938; 22: 1-10.

2 Haas J. Über das Zusammenvorkommen von Varanderungen der Retina und Choriodea. Arch Augenheilkd 1898; 37: 343-8.

3 Odland M. Congenital retinoschisis. Acta Ophthalmol (Kbh) 1981; 59: 649-58.

4 Juler F. An unusual form of retinal detachment (?cystic) in children. Trans Ophthalmol Soc UK 1948; 67: 199-205.

5 Sabates FN. Juvenile retinoschisis. Am J Ophthalmol 1966; 62: 683-8.

6 Levy J. Inherited retinal detachment. Br J Ophthalmol 1952; 36: 626-36.

7 Burns RP, Lovrien EW, Cibis AB. Juvenile sex-linked retinoschisis: clinical and genetic studies. Trans Am Acad Ophthalmol Otolaryngol 1971; 75: 1011-21.

8 Thomson E. Memorandum regarding a family in which neuroretinal disease of an unusual kind occurred only in the males. $\mathrm{Br} J$ Ophthalmol 1932; 16: 681-6.

9 Bengtsson B, Linder B. Sex-linked hereditary juvenile retinoschisis. Acta Ophthalmol (Kbh) 1967; 45: 411-23.

10 Sorsby A, Klein M, Gann JH, Siggins G. Unusual retinal detachment, possibly sex-linked. Br J Ophthalmol 1951; 35: $1-10$.

11 Hirose T, Schepens CL, Brockhurst, RJ, Wolf E, Tolentino FI. Congenital retinoschisis with night blindness in two girls. Ann Ophthalmol 1980; 12: 848-56.

12 Conway BP, Welch RB. X-chromosome-linked juvenile retinoschisis with hemorrhagic retinal cyst. Am J Ophthalmol 1977; 83: 853-5.

13 Kraushar MF, Schepens CL, Kaplan JA, Freemen HM. Congenital retinoschisis. In: Bellows JG, ed. Contemporary ophthalmology honoring Sir Stewart Duke-Elder, 1972. Baltimore: Williams and Wilkins, 1972: 265-90.

14 Hung JY, Hilton GF. Neovascular glaucoma in a patient with X-linked juvenile retinoschisis. Ann Ophthalmol 1980; 12: 1054-5.

15 Cibis PA. Retinoschisis-retinal cysts. Trans Am Ophthalmol Soc $1965 ; 63: 417-53$

16 Noble KG, Carr RE, Siegel IM. Familial foveal retinoschisis associated with a rod-cone dystrophy. Am J Ophthalmol 1978; 85: 551-7.

17 Forsius $\mathrm{H}$, Krause U, Helve J, et al. Visual acuity in 183 cases of X-chromosomal retinoschisis. Can J Ophthalmol 1973; 8: 385-93.

18 Richardson J. Juvenile retinoschisis, anterior retinal dialysis, and retinal detachment. BrJ Ophthalmol 1973; 57: 34-40.

19 Peyman GA, Green JL, Carroll CP. A simplified approach to the management of tangential retinal traction bands. $\mathrm{Br} J$ Ophthalmol 1981; 65: 414-6.

20 Deutman AF. Twenty vitreoretinal dystrophies. In: Krill AE, ed. Krill's hereditary retinal and chorodial diseases. Hagerstown: Harper and Row, 1977; 11: 1043-62.

21 Falls HF. Retinoschisis. Clinical description and course. In: Kimura SJ, Caygilla WM, eds. Retinal diseases. Philadelphia Lea and Febiger, 1966: 182-3.

22 Odland M. Congenital retinoschisis. Acta Ophthalmol (Kbh) 1981; 59: 649-58.

23 Bengtsson B, Linder B. Sex-linked hereditary juvenile retinoschisis. Presentation of two affected families. Acta Ophthalmol (Kbh) 1967; 45: 411-23.

24 Verdague TJ. Juvenile retinal detachment. Am J Ophthalmol 1982; 93: 145-56.

25 Yanoff M, Rahn EK, Zimmerman LE. Histopathology of juvenile retinoschisis. Arch Ophthalmol 1968; 79; 49-53.

26 Lisch W (translated by Blodi FC). Hereditary vitreoretinal degenerations: sex-linked juvenile retinoschisis, In: Straub W, ed. Developments in ophthalmology. New York: Karger, 1983: 19-32.

27 Bec P, Ravault M, Arne JL, Trepsat C. La péripherie du fond d'oeil. Paris: Masson, 1980.

28 Retina Society Terminology Committee. The classification of retinal detachment with proliferative vitreoretinopathy. Ophthalmology (Rochester) 1983; 90: 121. 